\title{
Refraction Static Correction without Picking First Arrival Times
}

\author{
Chien-Ying Wang ${ }^{1, *}$, Yi-Hen Lee ${ }^{1,2}$, and Jo-Pan Chang ${ }^{1,3}$ \\ 1 Institute of Geophysics, National Central University, Jhongli, Taiwan, ROC \\ ${ }^{2}$ Energy and Environment Research Laboratories, Industrial Technology Research Institute, Hsinchu, Taiwan, ROC \\ ${ }^{3}$ Center for General Education, Hsing Kuo University of Management, Tainan, Taiwan, ROC
}

Received 9 July 2008, accepted 25 December 2008

\begin{abstract}
A concept of differential delay time is proposed for refraction static correction without picking first arrival times in the $\mathrm{CDP}$ reflection data processing. This new method is a modification of the ABCD method; it uses cross-correlation to measure the first arrival time difference between signals received at stations $B$ and $C$, instead of directly computing them from their picked times. By taking advantage of multiple-fold CDP data, we apply the 'line-up trace' measurement of cross-correlations, which may alleviate the effect of data imperfections. The problem of refractor velocity variation has also been solved to a certain extent, which allows for a reliable delay time to be adequately estimated for each station and consequently the static correction value. A synthetic model and a real case with a severe weathered layer problem have been tested to evaluate the method. Stable and manageable computation processes have been explored to attain the maximum performance. The results are quite satisfactory. It should be possible to apply this method in rough areas with complicated refraction static problem, even in $3 \mathrm{D}$ cases.
\end{abstract}

Key words: Reflection seismics, Refraction seismics, Static correction, High-resolution

Citation: Wang, C. Y., Y. H. Lee, and J. P. Chang, 2009: Refraction static correction without picking first arrival times. Terr. Atmos. Ocean. Sci., 20, 769-778, doi: 10.3319/TAO.2008.12.25.01(T)

\section{INTRODUCTION}

Static correction is an important topic in reflection seismic investigations, especially for areas with rough surface conditions (Musgrave 1967; Cox 1999). This correction can be divided into two types: long-wavelength and short-wavelength statics. The long-wavelength static correction is accomplished with refraction statics and the short-wavelength by residual static corrections. This paper extends the $\mathrm{ABCD}$ refraction static method (ABCD means two sources at $A$ and $\mathrm{D}$ and two receivers at $\mathrm{B}$ and $\mathrm{C}$; Bahorich et al. 1982) to the treatment of the first arrival signals acquired in a CDP reflection survey. A differential delay time concept is proposed, which relies on a cross-correlation to measure the difference of the first arrival time (i.e., first-break) at two neighboring stations. Thus, the tedious process of first arrival time (FAT) picking is avoided.

Refraction static correction has been developed over the past 50 years (Yilmaz 2001). This correction technique takes

\footnotetext{
* Corresponding author

E-mail:wangcy@cc.ncu.edu.tw
}

parts of the first arrival signals of regular CDP reflection records, treating them as refracted waves traveling across the near-surface weathered layer. After picking the first arrival time, either the traditional refraction theory (Palmer 1981), least-squares inversion (Farrell and Euwema 1984; Hampson and Russell 1984; Taner et al. 1998), or tomography mapping (Chon and Dillon 1986; Docherty 1992) technique is applied to reveal the shape of the weathered layer, from which the static correction values are determined. Marsden (1993) gave a review of many methods prevailing in the refraction static field. Basically, the refraction static correction is used for removing first arrival time variations from the CDP records, making signals similar to travel through a homogenous surface layer. Hatherly et al. (1994) have suggested correcting them to a straight line, which represents a flat refractor after the correction. However, if the first arrivals of shot records were thought of as the refraction signals, it could seem more convincing to strictly stick to the refraction theory, the basis of refraction statics, to solve the problem (Yilmaz 2001). 
Most refraction static correction methods start at the first arrival time (FAT) picking, which is time-consuming and tedious. It is better if there are clear first breaks for shot records, and a certain degree of error is unavoidable if the recorded signals do not show sharp onsets. Moreover, the ability of judging the data quality may be lost, since only a time value is extracted from the first arrival waveform. Although there are many effective FAT automatic-picking algorithms, the users are always urged to check auto-picking results due to their imperfections. The method proposed in this paper, however, is designed to circumvent the FAT picking process and makes the method more objective.

Traditionally, the ABC method is commonly used in refraction static estimation, also known as the Plus-Minus method in the classical refraction theory (A and $\mathrm{C}$ are the shots while $\mathrm{B}$ in the middle is the receiver) (Hagedoorn 1959). In the ABCD method (Bahorich et al. 1982), an additional receiver, $\mathrm{C}$ is added and the difference of the FAT between $\mathrm{B}$ and $\mathrm{C}$ with the shots from the two sides, $\mathrm{A}$ and $\mathrm{D}$, is calculated. Eventually, this 'differential time' allows for probing of the more interesting visions of the potential usefulness of refraction signals (Cunnigham 1974; Chun and Jacewitz 1981; Lawton 1989). In the next sections, we will review the method, point out its weaknesses, and propose a workable solution that makes refraction static calculations more stable, reliable, and easier to apply.

\section{METHOD}

\subsection{Theory}

Figure 1 describes the geometry of the proposed method, which relies on the theory of 'delay time' (Barry 1967; Garner 1967). The concept is relatively simple. Although the refracted ray takes a slant path to incident upon a refractor layer (Fig. 1a), its travel time can be decomposed into the delay time $\left(\mathrm{Tz}_{\mathrm{A}}\right.$ and $\left.\mathrm{Tzj}\right)$ and the horizontal travel time (TX). The delay time is correlated to the depth by a 'depth conversion factor', DCF $\left(\mathrm{V}_{1} \mathrm{~V}_{2} / \sqrt{\mathrm{V}_{2}^{2}-\mathrm{V}_{1}^{2}}\right)$, where $\mathrm{V}_{1}$ and $\mathrm{V}_{2}$ are the velocities of the first (weathering) and the second (bedrock) layers, respectively. The velocity $\mathrm{V}_{1}$ of weathering layer is measured from the direct wave. The horizontal travel time, $\mathrm{Tx}$, is related to the horizontal distance simply by the refractor velocity, $\mathrm{V}_{2}$, as $\mathrm{Tx}=\mathrm{X} / \mathrm{V}_{2}$. However, when the refractor surface is tilted by an angle, $\delta$, a $\cos \delta$ term needs to be included into the distance changes. In the application, cos $\delta$ is always merged with the velocity $\mathrm{V}_{2}$ to $\mathrm{V}_{2} / \cos \delta$, similar to that for stacking velocity $\mathrm{V} / \cos \delta$ in the basic CDP theory for dipping layers. Following this concept, velocity of the refractor is a general term that accounts for horizontal travel time as well as variation of refractor shapes.

Using the concept of vertical time (or delay time) and horizontal time, we can easily establish the equations for the differential delay time method (Fig. 1). In fact, this is the
ABCD method proposed first by Bahorich et al. (1982); however, largely simplified here by means of 'delay time'. When the shot is on the left-hand side (Fig. 1b):

$\mathrm{Tzj}+\sum_{\mathrm{k}=\mathrm{i}}^{\mathrm{j}} \mathrm{T} \mathrm{x}_{\mathrm{k}}-\mathrm{Tzi}=\Delta \mathrm{Tji}$

When the shot is on the right-hand side:

$\mathrm{Tzi}+\sum_{\mathrm{k}=\mathrm{i}}^{\mathrm{j}} \mathrm{Tx}_{\mathrm{k}}-\mathrm{Tzj}=\Delta \mathrm{Tij}$

where $\Delta \mathrm{Tji}$ is the arrival time difference between stations $\mathrm{i}$ and $\mathrm{j}$ for the shot on the left and $\Delta \mathrm{Tij}$ for the shot on the right. Taking summation or subtraction of Eqs. (1) and (2), we get:

$\sum_{\mathrm{k}=\mathrm{i}}^{\mathrm{j}} \operatorname{Tx}_{\mathrm{k}}=(\Delta \mathrm{Tji}+\Delta \mathrm{Tij}) / 2$

Tzj $-\mathrm{Tzi}=(\Delta \mathrm{Tji}-\Delta \mathrm{Tij}) / 2$

$\Sigma$ Tx is the horizontal time that is composeds of several $\mathrm{x}$ elements between stations $\mathrm{i}$ and $\mathrm{j}$. However, $\mathrm{Tx}_{\mathrm{i}}$ is not to be calculated in detail, since several segments of the velocity $\mathrm{V}_{2 \mathrm{I}}$, or slowness $\mathrm{S}_{\mathrm{I}}$, along the line are sufficient to handle the velocity as well as the refractor shape variations, thus:

$\sum_{\mathrm{k}=\mathrm{i}}^{\mathrm{j}} \mathrm{Tx}_{\mathrm{k}}=\Sigma_{\mathrm{I}} \mathrm{S}_{\mathrm{I}} \Delta \mathrm{X}_{\mathrm{I}}=(\Delta \mathrm{Tji}+\Delta \mathrm{Tij}) / 2$

In these equations, the arrival time differences, $\Delta \mathrm{Tji}$ and

(a)

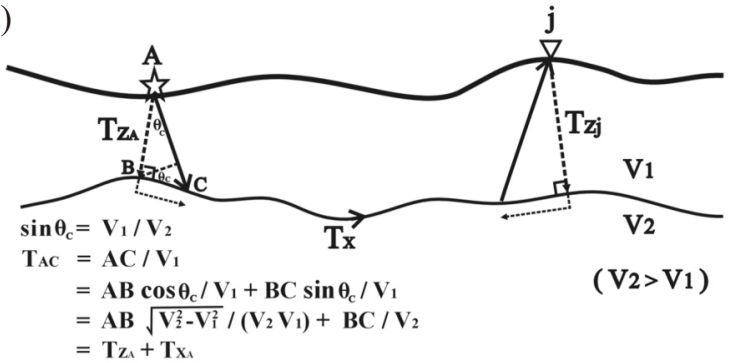

(b)

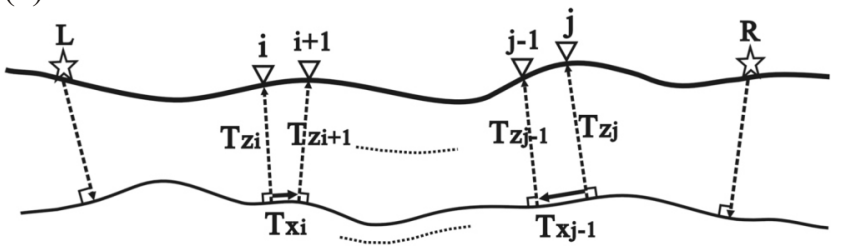

Fig. 1. A description of the theory of the differential delay time method (DDT): (a) shows that the travel time of the refracted ray path can be decomposed into two delay times $\left(\mathrm{Tz}_{\mathrm{A}}\right.$ and $\left.\mathrm{Tzj}\right)$ and one horizontal travel time (Tx). The equations show how the travel time decomposition is obtained; (b) illustrates the geometry of the DDT. The arrival time difference between stations $i$ and $j$ can be attributed to the difference between their delay time and the horizontal travel time in between. The shots ( $\mathrm{L}$ and $\mathrm{R}$ ) on both sides are needed. 
$\Delta$ Tij, can be calculated by comparing the refraction waveforms observed at stations $i$ and $j$ for shots from two different directions. Cross-correlation is no doubt the best function for evaluating the time difference. This will be discussed later. The suggested separation range between $i$ and $j$ should be kept within 10 locations to maintain their waveform similarities. The right hand sides of the Eqs. (4) or (5) are observed values. Equation (5) is applied first, which sets up a large matrix with the element positions appropriately assigned by station intervals, $\Delta X_{I}$ values. The matrix is then inverted to solve $\mathrm{S}_{1}, \mathrm{~S}_{2}, \ldots$ by using the standard singular value decomposition (SVD) method. Similarly, Eq. (4) is used to solve for $\mathrm{Tz}_{1}, \mathrm{Tz}_{2}, \ldots$, etc. The SVD method can smooth out the faulty data and makes the algorithm stable, when $\Delta \mathrm{Tji}$ and $\Delta \mathrm{Tij}$ are affected by errors. This SVD computation also makes the method fall into the category of least-squares inversion (Hampson and Russell 1984; Taner et al. 1998).

During computation, we first calculate Eq. (5) to find $\mathrm{S}_{\mathrm{I}}$ and then convert it to the velocity $\left(\mathrm{V}_{2 \mathrm{n}}=1 / \mathrm{S}_{\mathrm{n}}\right)$; thus this equation is related to a velocity equation. Note that the lateral varying $\mathrm{V}_{2}$ velocities are obtained as wished. Next, we use Eq. (4) to calculate the Tz's and convert them to the depths $(\mathrm{Zi}=\mathrm{Tzi} \times \mathrm{DCF})$. Equation (4) is thus the depth equation. Equations (4) and (5) are quite similar to the plus term and the minus term in the conventional Plus-Minus method (Hagedoorn 1959), or equivalent to the time-depth function and the velocity-analysis function in the generalized reciprocal method (Palmer 1981). These formulations take advantage of 'differential time' to concentrate the mapping under each location. Since the proposed method is based on the difference of two delay times, it is named the Differential Delay Time method (abbreviated as DDT).

It is noted that Eq. (4) is independent of Eq. (5), providing the advantages within the method. If we only want delay times and convert them to the static correction values by timing the $\mathrm{K}$ factor $\left[\sqrt{\left(\mathrm{V}_{2}-\mathrm{V}_{1}\right) /\left(\mathrm{V}_{2}+\mathrm{V}_{1}\right)}\right]$, assuming that $\mathrm{V}_{1}$ and $\mathrm{V}_{2}$ are known, we do not need Eq. (5). The $\mathrm{K}$ factor comes solely from the DCF timing $\left(1 / \mathrm{V}_{1}-1 / \mathrm{V}_{2}\right)$ (Chun and Jacewitz 1981). This takes into account the weathered layer correction, if the $\mathrm{V}_{1}$ layer is replaced by the $\mathrm{V}_{2}$ layer. Equation (5) (the velocity equation) is not necessarily required. A single $\mathrm{V}_{2}$ value is usually assumed in the generalized inversion calculation (Yilmaz 2001). This approach, however, may lead to problems on the two sides of the survey line. Equations (4) and (5) need shots from both sides (shooting reciprocally), but in this case we only have shots on one side for the side regions (see Fig. 3 for reference). At these places, we are forced to use Eqs. (1) and (2) to fill the gap. Here Eqs. (1) and (2) are modified by moving the $\Sigma T x$ term to the right-hand side, this term is evaluated by setting the proper $\mathrm{V}_{2}$ velocity. We will talk about this 'oneside-effect' in more detail when using the synthetic model in Fig. 3 to do the testing. A certain degree of smoothing is usually needed to lessen abrupt variations in the Tz's or $\mathrm{S}_{\mathrm{I}}$ 's.

\subsection{Cross Correlations}

With the exception of low-fold regions, many pairs of waveforms can be used to calculate one $\Delta \mathrm{Tij}$, for the station pair, $i$ and $j$. Figure 2 illustrates such a situation. The time difference between locations A and B can be estimated by using the refraction waveforms obtained from the entire shot records which have common receiver locations (Lawton 1989). These waveforms can be piled up to form two 'lineup traces'. The time difference $\Delta$ Tij is then calculated by measuring the cross-correlation of these two line-up traces, the maximum peak position will give the wanted time difference. The line-up trace has the advantage of suppressing ill-effects due to bad data pairs, and averages and smoothes cross-correlation computation.

In practice, however, we do not need to create line-up traces, but just sum up every individual cross-correlation (the results are the same). We may keep individual cross-correlations until the whole set has been created. The quality of each cross-correlation can be examined by analyzing their waveforms such as the deviation at each cross-correlation's peak time value or their similarity with each other. Bad cross-correlations can then be dropped in the line-up trace summation; this will make the final cross-correlation more reliable. This editing procedure has proven to be efficient in real data applications, such as the one to be shown in Fig. 6b.

\subsection{Absolute Level $\left(\mathrm{Tz}_{1}\right.$ Problem)}

We have calculated the delay time based only on their difference. A DC-level problem will arise if there is not a

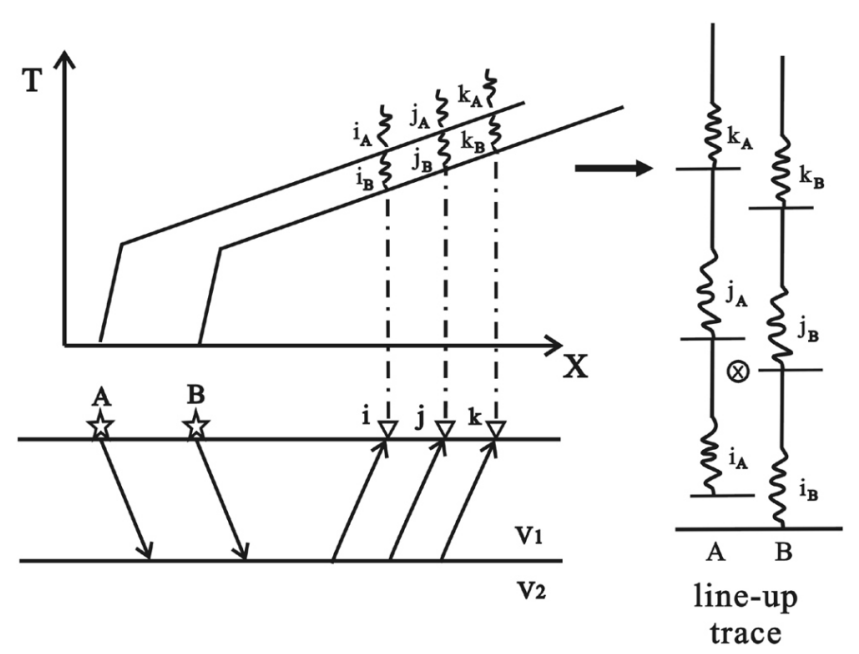

Fig. 2. The concept of multiple cross-correlation measurement. The arrival time difference between positions $\mathrm{A}$ and $\mathrm{B}$ can be obtained by comparing signals, $\mathrm{i}_{\mathrm{A}}$ and $\mathrm{i}_{\mathrm{B}}$, which are the first arrival parts received at the station $\mathrm{i}$ from shots A and B, respectively. Similar measurements can be obtained at stations $\mathrm{j}, \mathrm{k}$, and so on. These signals are thus appropriate for compiling into a line-up trace, and a more stable cross-correlation can be calculated by comparing these two line-up traces. 

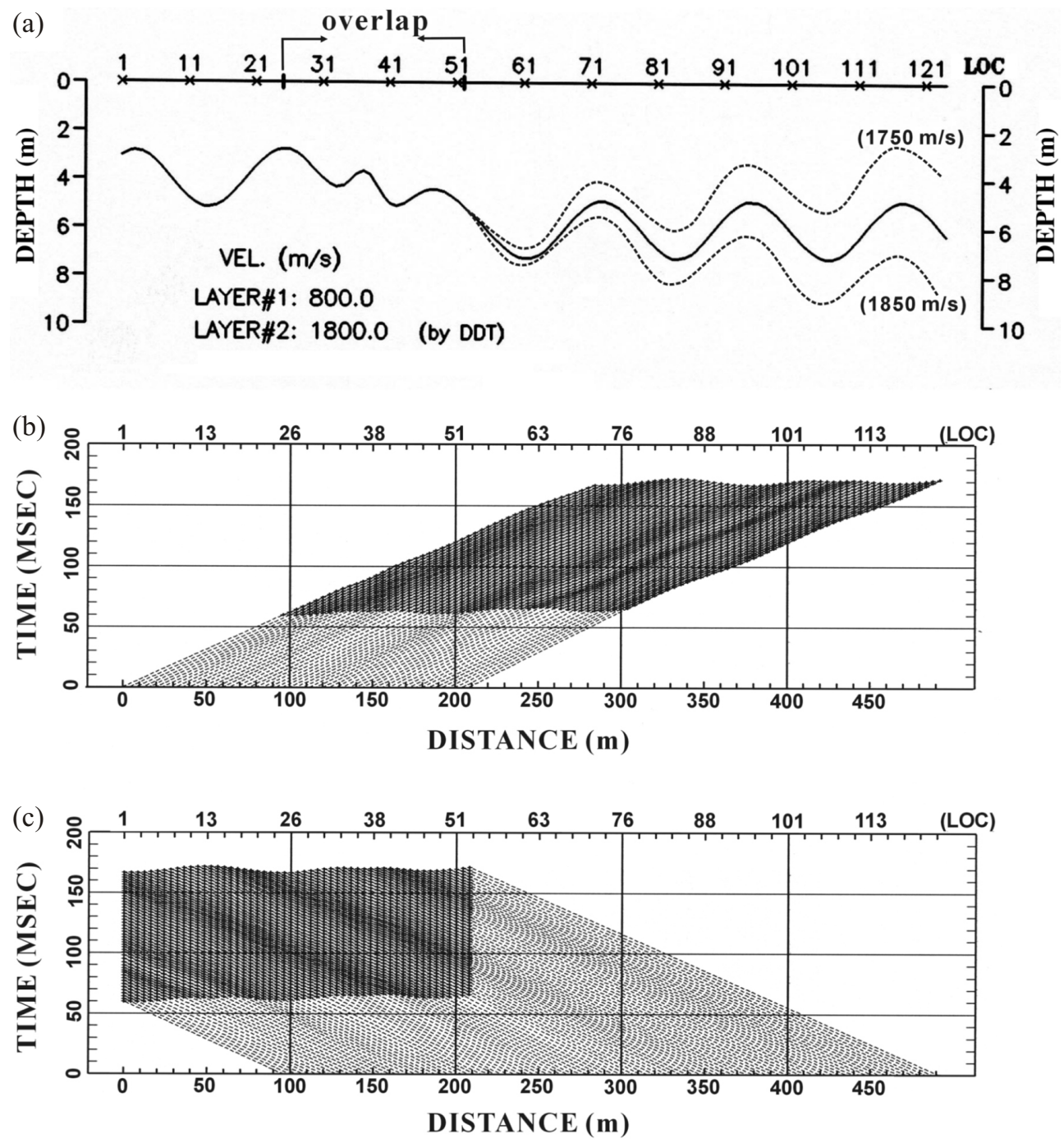

Fig. 3. (a) A sine-type undulating model for testing the DDT theory. Synthetic shot records of refracted waves are calculated using a CDP geometry: 4-m interval, 96-m near-offset, 48 channels, end-on shooting, 24 folds, and 53 shots. The theoretical first arrival times (FAT) are plotted in (b), which is then reciprocally mapped to simulate a shooting in the reverse direction (c). Only a small portion of the profile (locations \#25 - \#53) is overlapped by the two direction shooting. In (a), we also test the effect of velocities on the side-region where the shots are only from one direction.

single anchored $\mathrm{Tz}$ value (e.g., at the first receiver position). Here, it is called the $\mathrm{Tz} z_{1}$ problem. In setting up the matrix when applying Eq. (4), this absolute $\mathrm{DC}-$ level or $\mathrm{Tz}_{1}$ problem can be solved if a single line including a given $T z_{1}$ value is added to the matrix. However, even after calculations in this way, a reference to the $T z_{1}$ value is obtained, but not the final absolute $T z_{1}$ value. To overcome this shortfall, we need to measure several FAT values from the observed records to provide information about the absolute level. Here, we suggest to measure 5 to 10 FAT's, those are evenly distributed along the line, to establish the constraints. A guessed $\mathrm{Tz}_{1}$ is given first, and all Tzi's are found. The new $T z_{1}$ values can be estimated by the formula: $T z_{1}$ (new) = average of (observed FAT - calculated FAT) $/ 2+\mathrm{Tz}_{1}$ (old). This new $\mathrm{Tz}_{1}$ value is then used for the next iteration. The calculated FAT in the above formula is obtained by summing the Tzi values from the shot and the receiver locations and the Tx value in between. This $T x$ is calculated using a $V_{2}$ value simply by $\mathrm{X} / \mathrm{V}_{2}$. This suggests another way to estimate the velocity $\mathrm{V}_{2}$. It is attained as that several different $\mathrm{V}_{2}$ 's are assigned to do tests and the best $\mathrm{V}_{2}$ is chosen when $\Sigma$ (observed FAT - calculated FAT $)^{2}$ is minimized. We find that this is actually an appropriate way to determine another kind of $\mathrm{V}_{2}$ values. The $\mathrm{V}_{2}$ values obtained from Eq. (5) sometime suffer from great uncertainties. In practice, only several $\mathrm{V}_{2}$ values along the line are needed to guarantee the right Tzi or static correction values. The $\mathrm{V}_{2}$ values obtained from the $T z_{1}$ test can be used to double-check the $V_{2}$ velocities from the main search of Eq. (5).

For static correction, however, an exactly right $\mathrm{Tz}_{1}$ value is not so necessary. In the refraction static calculation, we need to adjust the static variations relative to a datum 
(referenced level), and the $\mathrm{Tz}_{1}$ can be treated as a datum. Relative Tzi values should be enough for removing the effect of irregular weathered layer.

\section{MODEL TEST}

A sine-shaped undulating refractor model (Fig. 3a) is used to test the DDT method. Velocities of the first and the second layers of this model are $\mathrm{V}_{1}=800 \mathrm{~m} \mathrm{sec}^{-1}$ and $\mathrm{V}_{2}=$ $1800 \mathrm{~m} \mathrm{sec}^{-1}$, respectively. A CDP survey is superimposed to create the synthetic records, using the following parameters: 4-m station interval, 96-m near-offset, 48 channels, 24 folds, end-on shooting, and 53 records to simulate a shallow reflection type of survey. A piece-wise refracted ray tracing algorithm (Spence et al. 1984) is then applied to calculate the refraction wave travel time. Figure $3 \mathrm{~b}$ compiles all the travel time curves from these 53 shots. In this shooting configuration, all shots are on the left-hand side of the receivers. By exchanging the source and the receiver locations (i.e., by using the reciprocal principle), we may simulate shooting in the reverse direction as that shown in Fig. 3c. These two shooting configurations are combined to produce an overlapping region between locations \#25 and \#53 (Fig. 3a). For those receivers inside the overlapped region, we have shots from both sides to form a stable portion, where Eqs. (4) or (5) can be applied. On the other hand, the regions on the left and the right sides have shots from only one direction. Equations (1) or (2) are used in these side regions.

53 synthetic shot records are generated by putting minphase Ricker wavelets at the time positions predicted in Figs. $3 \mathrm{~b}$ and c. Figure $4 \mathrm{a}$ describes some samples. The refracted waveforms are selected from these records and crosscorrelations are taken to measure the difference of the first arrival time, $\Delta \mathrm{Tij}$ or $\Delta \mathrm{Tji}$. Figure $4 \mathrm{~b}$ shows two cases, one for a shot pair (\#5 and \#8) and the other for a receiver pair (\#30 and \#33). The numbers of available cross-correlations for these two cases are different, however, both of them can be used to measure the time difference between the positions ' $i$ ' and ' $j$ '. The resulting cross-correlations are indicated at the bottom row of Fig. 4b. The time difference is determined by the time position at the maximum peak of the calculated cross-correlation. However, the second and the third peaks could also provide $\Delta$ Tij information if the paired waveforms are not consistent enough to prevent possible cycle skips (i.e., peak miss-picking). The cycle skip problem can be partially solved by comparing the calculated $\Delta$ Tij and the observed $\Delta$ Tij after the refractor model has been established.

After determining the $\Delta$ Tij's, we enter the DDT procedures by applying Eq. (4) for the overlapping region and the modified Eqs. (1) or (2) ( $\Sigma$ Tx term being estimated using given $\mathrm{V}_{2}$ 's) for the side regions. A paradox may arise in the side regions, since the refractor dip angle cannot be evalu-

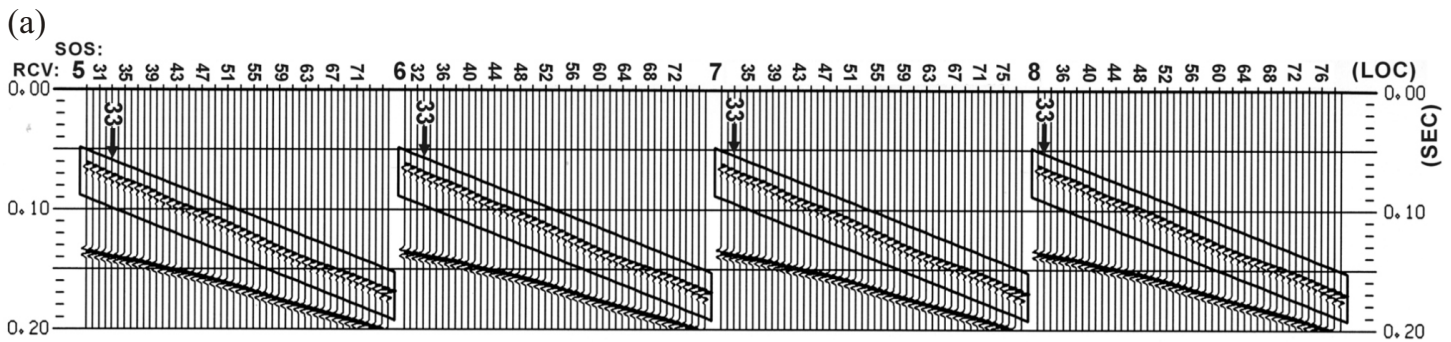

(b)

common-shot

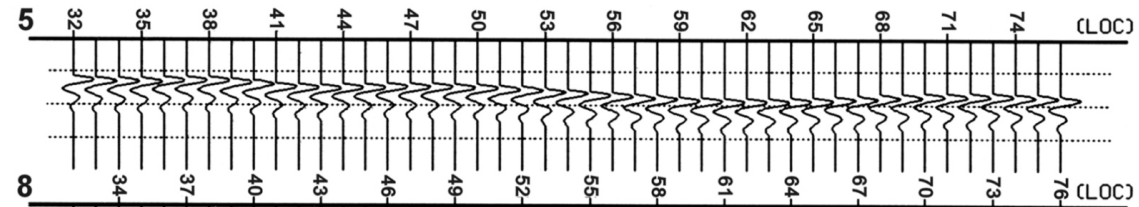

8

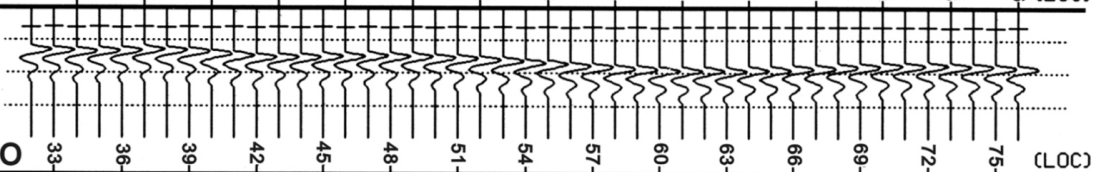

CRO

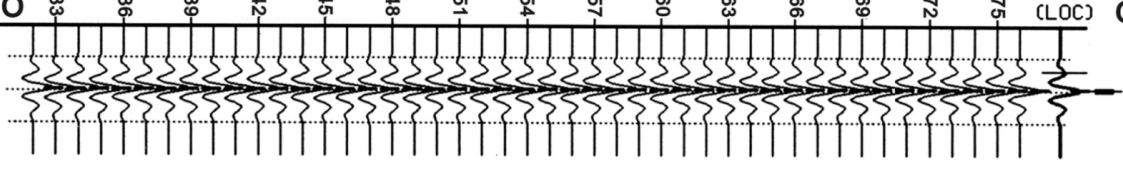

common-receiver
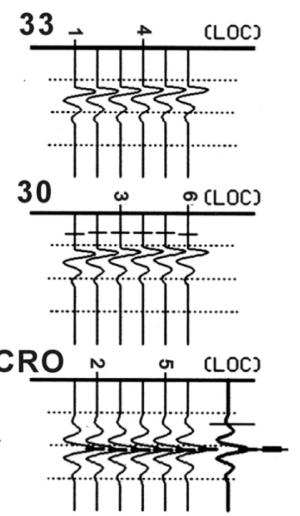

Fig. 4. (a) shows the synthetic shot records of refracted waves based on the model in Fig. 3a. The refracted waves are selected as indicated by the bracket. (b) displays the selected refracted waveforms and their cross-correlations (CRO). The left and right parts are for the common-shot and the common-receiver, respectively. The number at the left-hand corner of the left part is the source location \# and the other numbers are the receiver locations. The summed cross-correlation is shown at the low-right corner. 
ated by using only one-direction shooting data. Nevertheless, we mentioned that the refractor dip angle can be absorbed into the velocity $\mathrm{V}_{2}$, i.e., $\mathrm{V}_{2} / \cos \delta$. Figure $3 \mathrm{a}$ shows the test for two other velocities, 1750 and $1850 \mathrm{~m} \mathrm{sec}^{-1}$, instead of the correct velocity, $1800 \mathrm{~m} \mathrm{sec}^{-1}$, on the right-end side. Tilted refractors are obtained when the $\mathrm{V}_{2}$ velocity is biased. On the other hand, if an 'averaged flat' refractor surface is assumed $(\delta=0)$, an appropriate $\mathrm{V}_{2}$ velocity can be estimated. This is another way, also efficient, to determine the pertinent $\mathrm{V}_{2}$ on the side regions. In fact, since Eq. (5) does not have the ability to provide the $\mathrm{V}_{2}$ velocity in the nonoverlapping region, the flat or any tilt (with a known dip angle) refractor assumption can be applied to determine good $V_{2}$ values for the side regions. The refractor slope and the velocity are associated with each other. This is actually the basic reason for all algorithms which do not require shots on both sides (Cunningham 1974).

In this test case, the DDT calculation gives nearly perfect refractor shape as the original model shown in Fig. 3a. Misfits mostly come from the $\mathrm{V}_{2}$ bias. Although Eq. (5) gives a reliable $V_{2}$, a shift may occur if the refractors undulate widely (i.e., $\cos \delta$ problem). This is, in fact, an inherent, theoretical weak point in all refraction statics methods. We need alternative ways to measure the $V_{2}$ such as the ones mentioned before, i.e., the $\mathrm{Tz}_{1}$ test or the averagedflat refractor assumption.

\section{FIELD CASE}

This section deals with the DDT method being applied to real data. A special case with a severe weathered layer correction problem is purposely chosen. Figure 5 shows the surface elevation variation and the refractor obtained from the DDT method. It is known that the underground layers in this area are relatively flat from drilling. The highly rugged surface makes the CDP stack image poor in this hilly area (see Fig. 8a). In order to examine the performance of DDT, we do not take any elevation correction, but include all near-surface corrections in the DDT processes.

The distorted first arrival time pattern and the waveforms can be seen in Fig. 6a, where the earlier arrivals of four consecutive shot records are displayed. It is obvious that FAT picking could be difficult with this kind of data. The refraction signals have been carefully extracted from these records and the selected waveform set is then processed using the DDT method. Figure $6 \mathrm{~b}$ shows the refraction waveforms (from shot locations \#2 and \#4) and their cross-correlation. Some bad cross-correlations are removed based on their peak time deviation and bad waveform similarity. They are marked by the ' $x$ ' symbols in the bottom row of Fig. 6b. The other good cross-correlations are then summed to form the final cross-correlation, which gives the final $\Delta \mathrm{T}_{24}$ value. This example highlights one merit of the DDT method, by using the waveform to control the data quality. This is especially important in a region with poor data such as in this case.

Figure 5 shows the refractor shape resulting from the DDT processing. Note that the 'overlapping' zone is quite narrow, just between locations $\# 25$ and $\# 53$. A gross $V_{2}$ velocity $\left(1600 \mathrm{~m} \mathrm{sec}^{-1}\right)$ is obtained from the $\mathrm{Tz} \mathrm{z}_{1}$ test and a $1800 \mathrm{~m} \mathrm{sec}^{-1}$ velocity is assigned to the right-side region to force the refractor shape following the trend determined in the overlapped zone. It is interesting to see that the refractor (bed rock) approaches the ground surface near locations \#10 to \#20. This bedrock exposure is confirmed by an outcrop along a near-by stream in the area. The greatest thickness of the weathered layer is about $40 \mathrm{~m}$, which may give static shift values as large as $100 \mathrm{msec}$. This is surely a large value for a shallow reflection survey. The CDP geometry of this real case is the same as that in the previous synthetic model test. We have purposely made these two tests (real and theoretically) compatible.

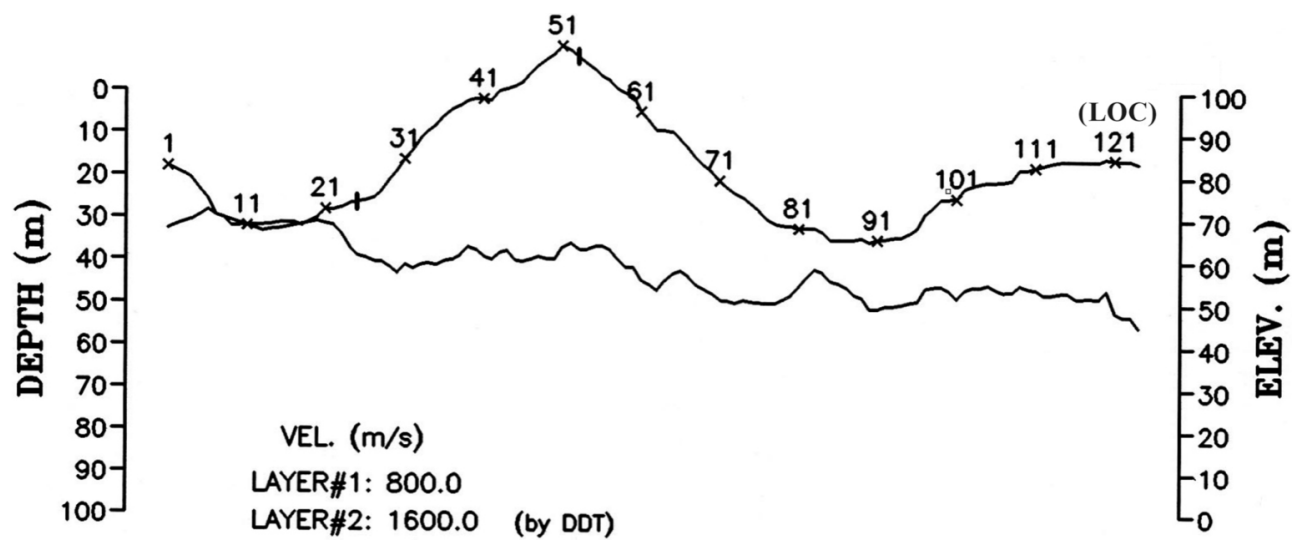

Fig. 5. A real case, with a severe weathered layer problem. The rough surface topography causes large static shifts, which can be as high as $100 \mathrm{msec}$. The datum has been set at the elevation $100 \mathrm{~m}$. The bed-rock surface mapped by DDT comes out relatively regular. The bed-rock outcrop between locations \#10 and \#20 has been confirmed by a field examination. 
(a)

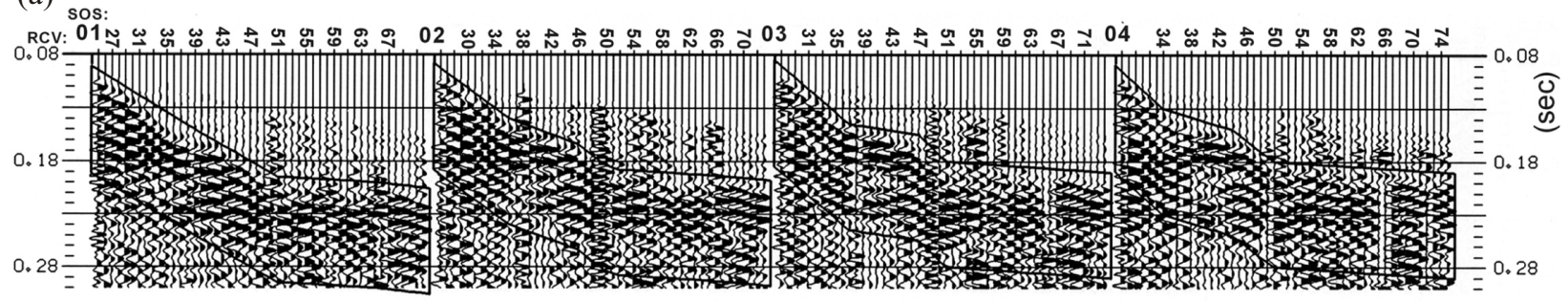

(b)

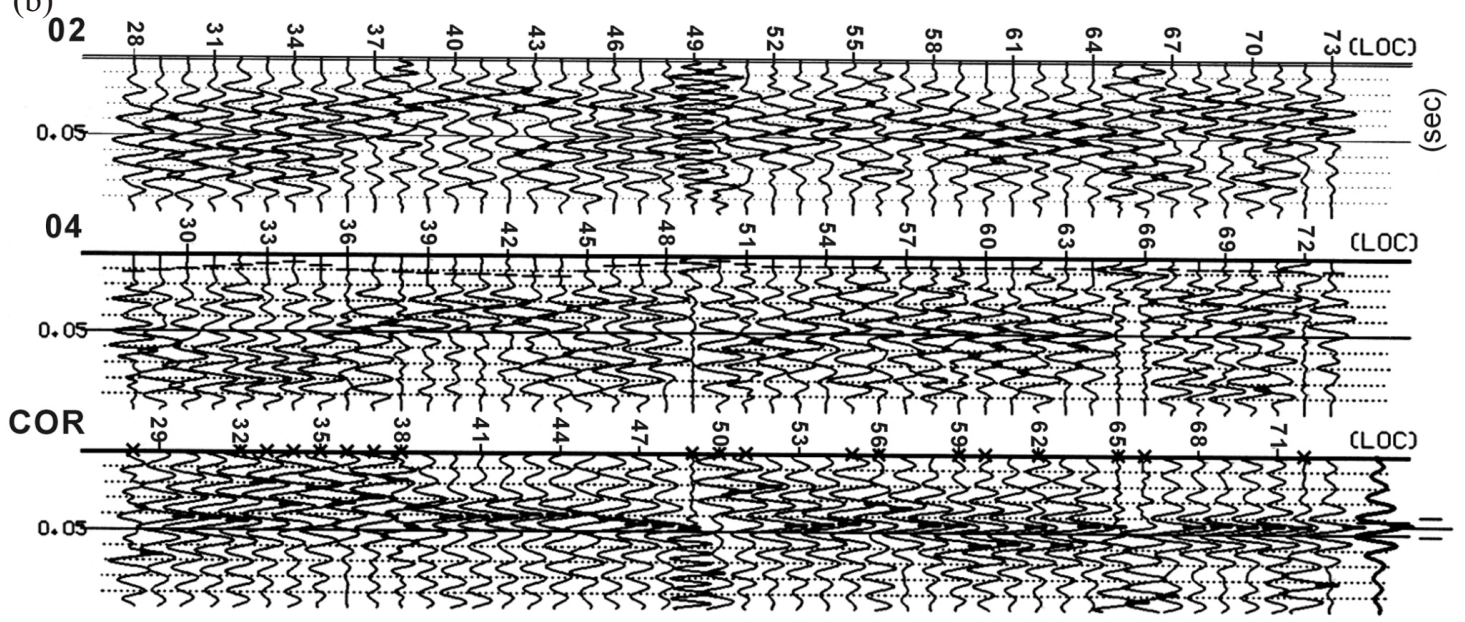

Fig. 6. (a) shows four observed shot records. The highly irregular first arrivals imply complicated near-surface conditions. The refracted waveforms to be analyzed have been bracketed. (b) shows the related refracted waveforms and their cross-correlations. Some bad data are excluded (indicated by cross signs) when calculating the cross-correlation.

The calculated refraction static correction is then applied to the original data set, and several residual static correction processes are followed to tune up the short-wavelength static variation. Figure 7 shows a comparison of a shot record before and after the refraction static correction. A dip-filter to remove the air-wave and a frequency-filter to restrict the signals within the $60-200 \mathrm{~Hz}$ band have been applied for this shot record before the DDT processing. It is surprising to find that the reflection events begin to line-up after the refraction static correction. Large static shifts are visible, such as those implied at the bottom of Fig. 7b. The aligned reflection signals in Fig. $7 \mathrm{~b}$ provide the opportunity to get better CDP stacking, as shown in Fig. 8. It is again a great surprise to check the final stacked section (Fig. 8b) after the application of static corrections. There is almost nothing in the first section of Fig. 8, but numerous reflection events emerge after static corrections. The event can even be seen down to a depth near the two-way time of $0.8 \mathrm{sec}$. The flat nature of the layers is consistent with the local geological investigations. This case indeed indicates the importance of the static correction for data collected in an area with rough surface conditions.

\section{DISCUSSIONS AND CONCLUSIONS}

The difficulty of picking first arrival times is bypassed in the DDT method proposed here. All refraction waveforms are involved in the computation, rather than a single FAT value. This provides a wider platform for using first arrival signals. The method takes advantage of the multi-fold nature of CDP, and tries to enhance the data quality by using multiple cross-correlations, which is actually similar to the powerful stacking procedure in the CDP technology. This should be a faithful way to use first arrival signals.

Although the tests carried out in this paper used an endon geometry, the method should work equally well, or even better, for a split-spread case. The overlapping zones are the main places where the DDT method is applied and the splitspread is supposed to provide more such places. The cases discussed here are for shallow reflection seismics, for which static correction is more urgently needed (Wang 2002) than in large-scale exploration seismics. However, there is no reason that the method could not work in different survey sizes.

The velocity may cause some trouble. If the refractor is smooth and relatively flat, the problem of the dip angle, i.e., $\cos \delta$, may not be serious. However, the fluctuations in real data can easily influence the slowness $\left(\mathrm{S}_{\mathrm{I}}\right)$ calculation, which is then magnified, as it is the denominator term in the velocity formula, i.e., $V=1 / \mathrm{S}$. This makes the $\mathrm{V}_{2}$ estimation, using Eq. (5), not as efficient for real data. However, the exact velocity values are not so important if only the static correc- 
tion values are wanted. The velocities affect just the $\mathrm{K}$ factor, which is used to convert the Tz values to the static correction values. The error percentage that affects the $\mathrm{K}$ value from $\Delta \mathrm{V}_{1}$, is $\mathrm{V}_{2} \Delta \mathrm{V}_{1} /\left(\mathrm{V}_{2}^{2}-\mathrm{V}_{1}^{2}\right)$, and from $\Delta \mathrm{V}_{2}$, is $\mathrm{V}_{1} \Delta \mathrm{V}_{2} /\left(\mathrm{V}_{2}^{2}-\mathrm{V}_{1}^{2}\right)$. Thus, a $10 \%$ error in $\Delta \mathrm{V}_{1}$ or $\Delta \mathrm{V}_{2}$ should affect the $\mathrm{K}$ factor by less than $10 \%$ for reasonable $\mathrm{V}_{1}$ and $\mathrm{V}_{2}$ values. The $\mathrm{Tz}$ values are basically determined independent of the velocity [Eq. (4)]. Nevertheless, the DDT method still proposes several ways to estimate the velocity. The most interesting one comes from the $\mathrm{Tz}_{1}$ test. By using limited FAT observations, we may constrain the $\mathrm{V}_{2}$ velocity values along the line at some acceptable level.
In this paper, we have emphasized on using cross-correlations to calculate first arrival time differences, however, it does not exclude that these time differences may simply come from the FAT, if they have already been picked. The significant point of DDT is to explore the maximum performance of time differences, like the plus term (a kind of delay time) in the traditional Plus-Minus method, not just how to calculate the time difference. Moreover, if we have plenty of FAT's, a more stable $\mathrm{Tz}_{1}$ kind of velocity analysis can be reached.

Due to the independence of the Tz calculation in this algorithm, it should be a robust procedure for focusing the (a)

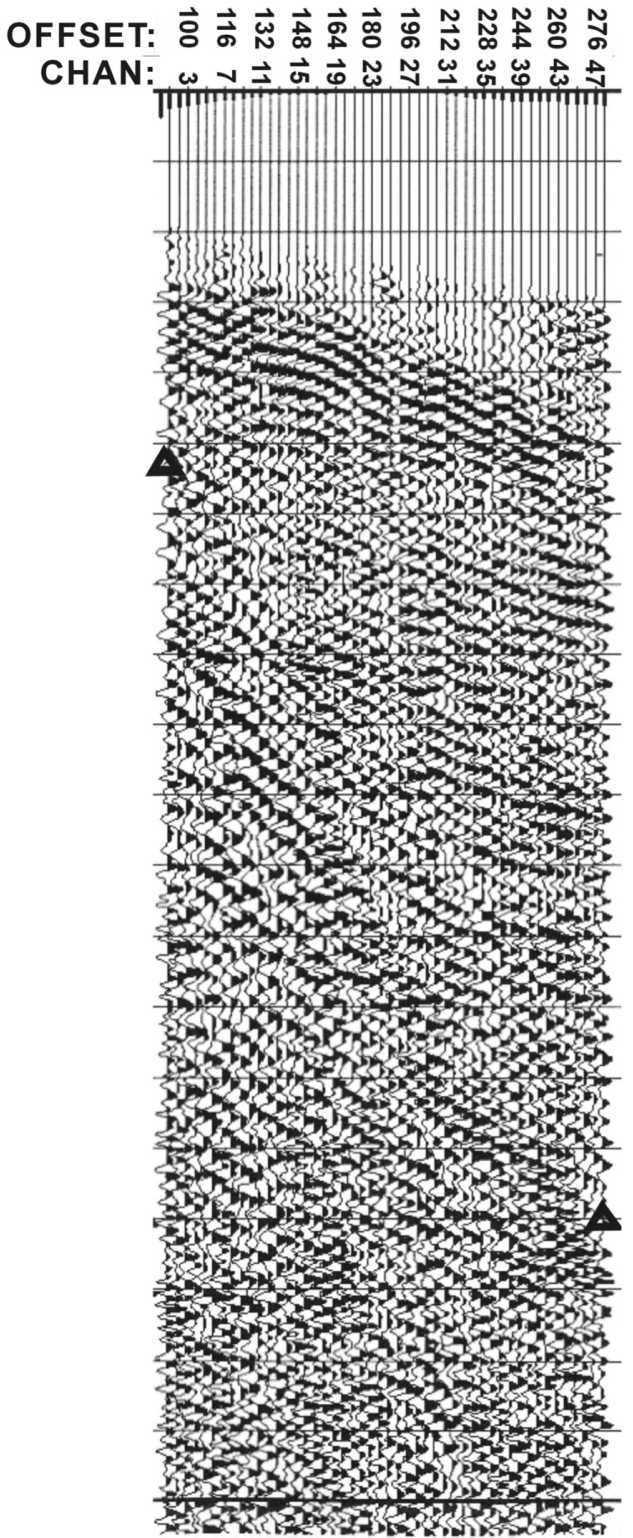

(b)
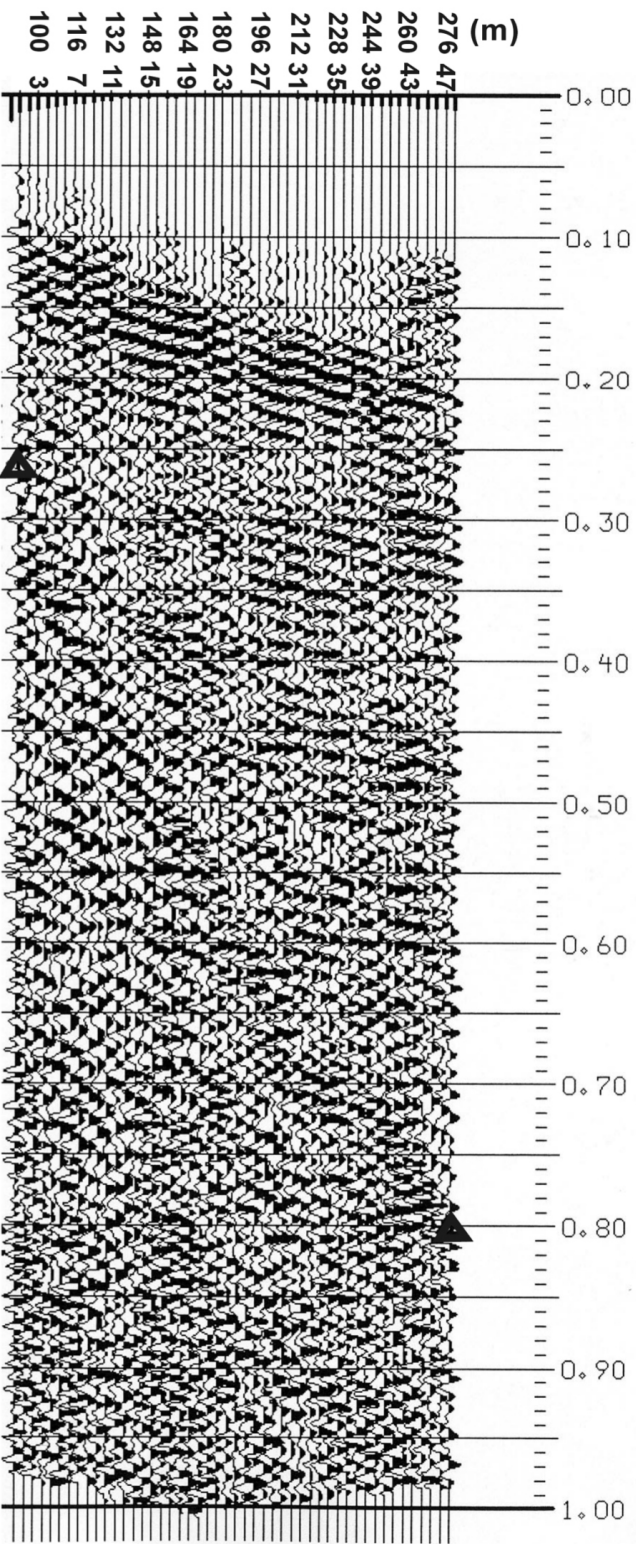

Fig. 7. An example of the shot record: (a) is before the DDT processing, on which some dip-filters and frequency-filters have been applied; (b) shows the result after the DDT refraction static correction. Some useful signals start to line-up, which leads to an effective stacked section shown in Fig. 8b. The triangles indicate the air wave arrival. 
(a)

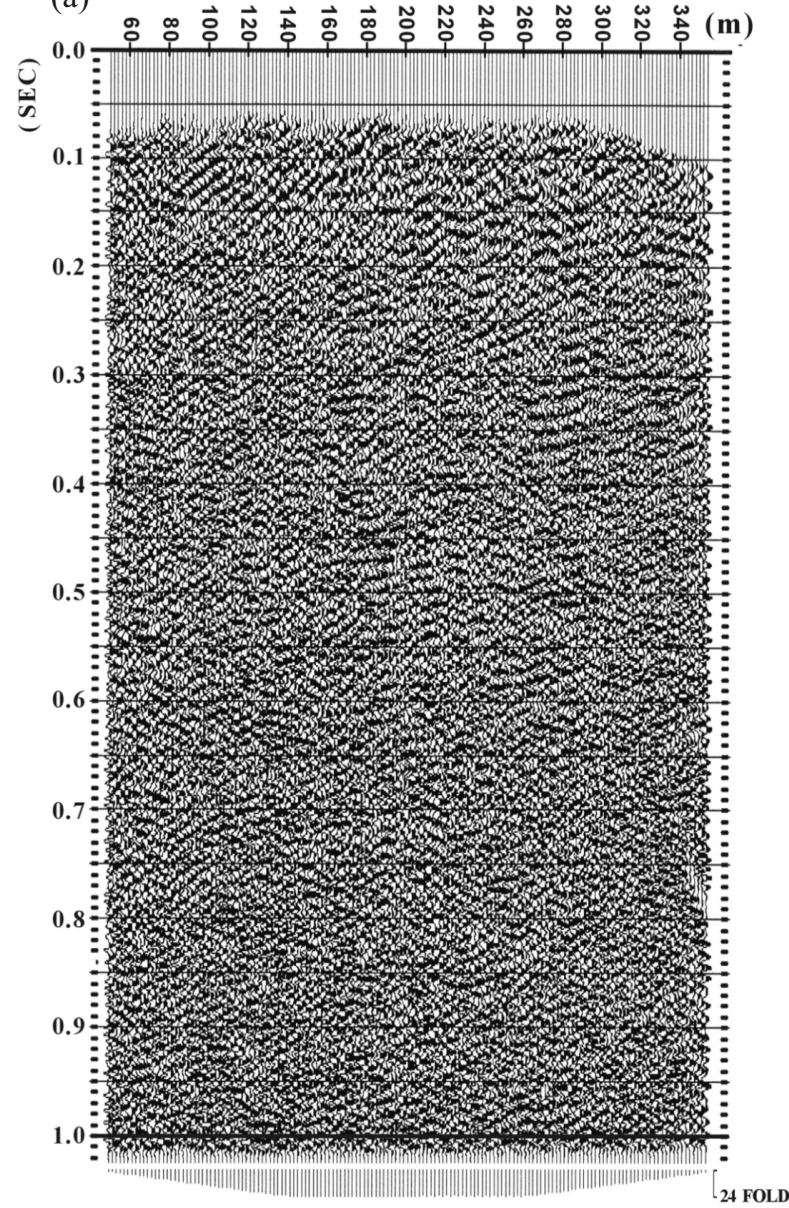

(b)

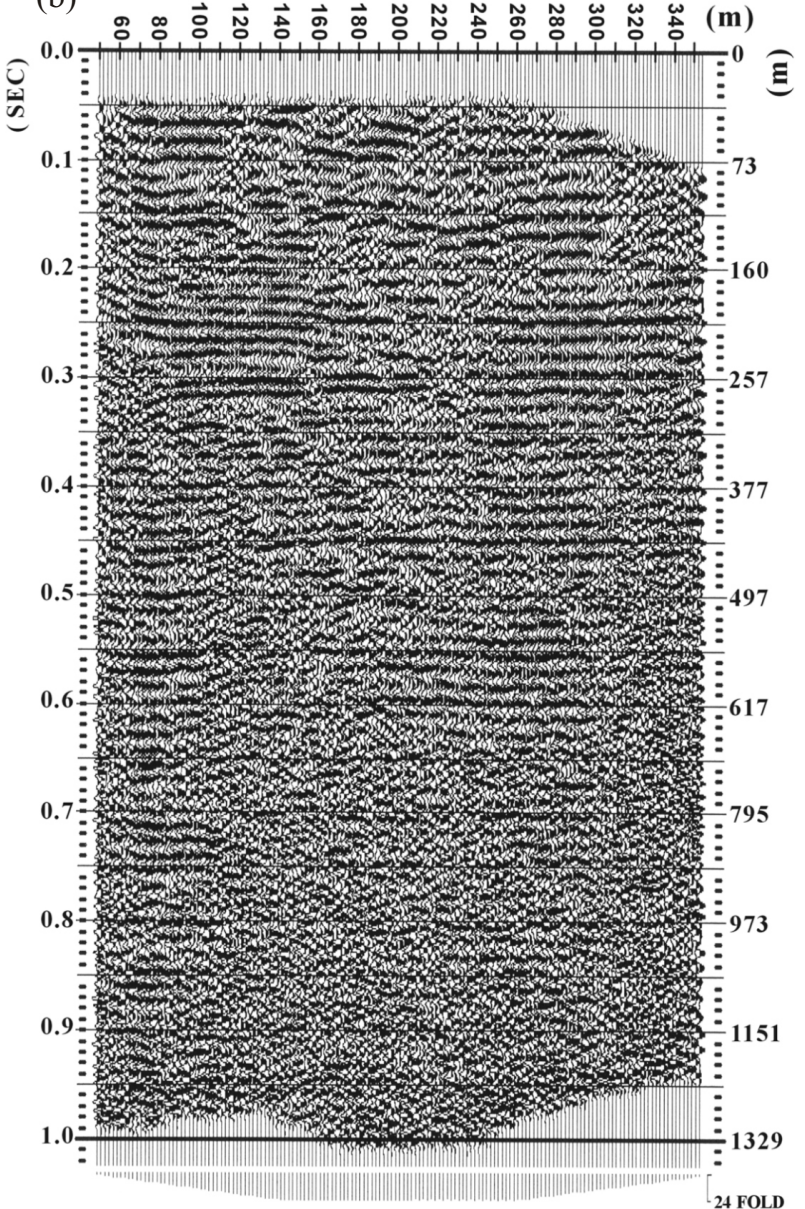

Fig. 8. A comparison of final sections: (a) without and (b) with DDT static corrections. Many flat events emerge after applying a large amount of static correction, which can be seen at the bottom of (b).

refraction static correction value at every location if the velocities have been controlled within a reasonable range. The basic reasoning for DDT is that it uses differential arrival times observed at the station pair under consideration, due to the shots from two sides, to 'cancel out' the parts in common. The information needed for each station to calculate static corrections is thus isolated. Besides its use in treating data from difficult surface conditions, we consider this method also to be an efficient way to calculate the nearsurface static values for 3D data (Taner et al. 1998). Ample data from the 'two' sides would be required to be used in the cancellation of the common path allowing the station statics to be reliably determined. This approach is worth future testing.

Acknowledgements This research was supported by the National Science Council of Taiwan under grant NSC 952116-M-008-001. We would like to show our sincere appreciation to the reviewers for their valuable suggestions which improved the paper.

\section{REFERENCES}

Bahorich, M. S., C. Coruh, E. S. Robinson, and J. K. Costain, 1982: Static correction on the southeastern Piedmont of the United States. Geophysics, 11, 1540-1549, doi: 10.1190/ 1.1441304. [Link]

Barry, K. M., 1967: Delay time and its application to refraction profile interpretation. In: Musgrave, A. W. (Ed.), Seismic Refraction Prospecting, Soc. Expl. Geophys., 348-361.

Chon, Y. T. and T. J. Dillon, 1986: Tomographic mapping of the weathered layer. $56^{\text {th }}$ Ann. Internat. Mtg., Soc. Expl. Geophys., Expanded Abstracts, 593-595.

Chun, J. H. and C. A. Jacewitz, 1981: The first arrival time surface and estimation of statics. $51^{\text {st }}$ Ann. Internat. Mtg., Soc. Expl. Geophys., Expanded Abstracts, 386-388.

Cox, M., 1999: Static Corrections for Seismic Reflection Surveys. In: Scherrer, E. F. and R. Chen (Eds.), Geophysical References, Volume 9, Society of Exploration Geophysicists, $531 \mathrm{pp}$.

Cunningham, A. B., 1974: Refraction data from single-ended refraction profiles. Geophysics, 39, 292-301, doi: 10.1190/ 
1.1440428. [Link]

Docherty, P., 1992: Solving for the thickness and velocity of the weathering layer using 2-D refraction tomography. Geophysics, 57, 1307-1318, doi: 10.1190/1.1443198. [Link]

Farrell, R. C. and R. N. Euwema, 1984: Refraction statics. Proc. IEEE, 72, 1316-1329, doi: 10.1109/PROC.1984.13020. [Link]

Garner, L. W., 1967: Refraction seismogram profile interpretation. In: Musgrave, A. W. (Ed.), Seismic Refraction Prospecting, Soc. Expl. Geophys., 338-347.

Hagedoorn, J. G., 1959: The plus-minus method of interpreting seismic refraction sections. Geophys. Prospect., 7, 158182, doi: 10.1111/j.1365-2478.1959.tb01460.x. [Link]

Hampson, D. and B. Russell, 1984: First break interpretation using generalized linear inversion. J. Can. Soc. Expl. Geophys., 20, 40-54.

Hatherly, P. J., M. Urosevic, A. Lambourne, and B. J. Evans, 1994: A simple approach to calculating refraction statics corrections. Geophysics, 59, 156-160, doi: 10.1190/1. 1443527. [Link]

Lawton, D. C., 1989: Computation of refraction static correc- tions using first-break travel time difference. Geophysics, 54, 1289-1296, doi: 10.1190/1.1442588. [Link]

Marsden, D., 1993: Static correction - a review, Part II. The Leading Edge, 2, 115-120.

Musgrave, A. W. (Ed.), 1967: Seismic Refraction Prospecting, Soc. Expl. Geophys.

Palmer, D., 1981: The generalized reciprocal method of refraction seismic interpretation. Geophysics, 46, 15081518, doi: 10.1190/1.1441157. [Link]

Spence, G. D., K. P. Wittal, and R. M. Clowes, 1984: Practical synthetic seismograms for laterally varying media calculated by asymptotic ray theory. Bull. Seismol. Soc. Am., 72, 5277-5217.

Taner, M. Y., D. E. Wagner, E. Baysal, and L. Lu, 1998: A unified method for 2-D and 3-D refraction statics. Geophysics, 63, 260-274, doi: 10.1190/1.1444320. [Link]

Wang, C. Y., 2002: Detection of a recent earthquake fault by the shallow reflection seismic method. Geophysics, 67, 1465 1473, doi: 10.1190/1.1512791. [Link]

Yilmaz, O., 2001: Seismic Data Analysis, $2^{\text {nd }}$ edition, Soc. Expl. Geophys. 